\title{
Ein doppeltes Plädoyer für internationale Öffnung und stärker vernetzte Interdisziplinarität
}

Stefan Grundmann

2014-06-30T16:19:27

Der Wissenschaftsrat ruft in seinem Gutachten zu den "Perspektiven der Rechtswissenschaft in Deutschland" dazu auf, die Rechtswissenschaft sowohl international als auch mit Blick auf die Nachbarwissenschaften zu öffnen. Beide Forderungen verdienen dann Unterstützung, wenn sie als Weiterentwicklung, nicht als revolutionäre Forderung zum Umsturz des bestehenden Systems verstanden werden.

\section{Hauptthesen des Gutachtens}

Die beiden Kernthesen des Gutachtens des Wissenschaftsrats zu den "Perspektiven der Rechtswissenschaft in Deutschland" gelten für Forschung und Lehre zugleich:

Ausgehend von der Erkenntnis, dass Rechtswissenschaften durch die „enge Theorie-Praxis-Verklammerung“ geprägt sind („Professionsfakultäten“) (S. 5 und 7), und getragen von einem positiven Grundtenor dahingehend, dass diese Verklammerung in Studium und Forschung auch grds. gut gelingt, trägt der Wissenschaftsrat ein Hauptpetitum vor und benennt er einen Hauptgrund dafür, dass die Rechtswissenschaft derzeit auf „beträchtliche Veränderungen ihres Gegenstandes“ zu reagieren habe: Hauptpetitum ist „die Stärkung der Grundlagenfächer, die Intensivierung des interdisziplinären wie disziplinären Austausches und eine Öffnung der Rechtswissenschaft in die Universität wie in das Wissenschaftssystem." (S. 7, näher S. 40 f.). Als Hauptgrund für beträchtliche Veränderungen im Gegenstand führt der Wissenschaftsrat „Prozesse der Europäisierung und Internationalisierung [an] sowie ... weitere [unbenannte] Strukturveränderungen des Rechts." (S. 5, etwas breiter S. 7). Institutionell mahnt der Rat eine stärkere Ausdifferenzierung an - vor allem eine größere Präsenz von Frauen, von ausländischen Lehrenden und eine Auffächerung der curricula.

Beide Hauptstoßrichtungen überzeugen in hohem Maße, wenn sie als Anregung zur Fortentwicklung verstanden werden und nicht als Absage an die bisherige Grundanlage. Dahingehend ist das Gutachten (wohl) auch zu verstehen.

Richtig ist auch, dass Veränderungen nicht nur inhaltlich, sondern auch mit institutionell-systemischen Mitteln zu fördern sind, um die Realisierungschancen zu erhöhen. Daneben steht freilich die inhaltliche Arbeit an den Veränderungen, aus der Forschung und Lehre heraus, und diese scheint mir umgekehrt für beide Hauptstoßrichtungen im Gutachten zu wenig gewürdigt zu sein. Konkret: Für beide Hauptpetita und -stoßrichtungen mag benannte Auffächerung wichtig sein, es existieren jedoch in Forschung und Lehre bereits wichtige Modelle. Ein zentrales 
Anliegen wäre daher deren allgemeinere Verbreitung und Unterstützung, weniger Neukonzeptionierung.

\section{Zur Forschung}

Der Abschnitt zur Forschung (und Publikationstätigkeit) ist noch stärker allein auf die institutionellen Maßnahmen fokussiert.

Betont wird im Ausgangspunkt die Bedeutung der Grundausstattung, die für die Forschung in kleinen Einheiten als zentral angesehen wird (S. $38 \mathrm{ff}$.). Dem ist umfassend zuzustimmen. Individuelle Forschung ist in den Rechtswissenschaften noch immer überragend wichtig, wohl ungleich mehr als in vielen anderen Disziplinen (und dies übrigens auch in den USA). Die Grundausstattung ist jedoch darüber hinaus häufig auch der Grundstock für Vernetzungen. Diese Feststellung legt freilich ordentlich ausgestattete Lehrstühle zugrunde. Die erhebliche Reduktion der Grundausstattung in den letzten Dekaden wäre noch viel entschiedener zu kritisieren gewesen. Sie bildet strukturpolitisch - neben der Aufblähung der Evaluations- und Verwaltungsaufgaben - den größten Sündenfall, jedenfalls für die Rechtswissenschaften. Die Grundlagen für die individuelle Forschung - Grundausstattung und Zeit - zu erhalten und dies für eine große Zahl an Rechtswissenschaftlern und Rechtswissenschaftlerinnen, ist, obwohl ich den beiden Hauptstoßrichtungen umfassend zustimme, ein ebenso zentrales Petitum.

Ebenfalls richtig ist das Petitum, dass in der Forschung die eigene Sprache zu erhalten ist - ich würde ergänzen: schon um die Vernetzung mit der notwendig deutschsprachigen Praxis zu erhalten -, dass jedoch eine europaweite oder globale Wahrnehmung eine intensivierte Publikationstätigkeit in Englisch (und anderen Sprachen) voraussetzt (S. 9 et passim). Das Petitum für Mehrsprachigkeit ist daher - hält man beide Arten der Vernetzung für wichtig - aus Sicht der Rechtswissenschaften in Deutschland alternativlos. Drei Aspekte scheinen mir hier zentral: Auch in dieser Hinsicht ist eine gute Grundausstattung wichtig und hilfreich. Da Mehrsprachigkeit im Oeuvre bei Evaluationen, vor allem aber Berufungen noch immer kaum ins Gewicht fällt, wären hier Leitlinien im Sinne der vom Wissenschaftsrat vorgeschlagenen „Bewertungsverfahren für Forschungsqualität" erwägenswert (S. 8, 38 ff.). Dass ein Beitrag in der Modern Law Review weniger wiegen soll als ein Beitrag in der Juristenzeitung, ist - trotz großer Hochachtung für die Juristenzeitung - ein Skandalon. Was dann die Stärkung der internationalen Einbettung und Vernetzung angeht, sind die mahnenden Worte des Wissenschaftsrats (S. 9, 42 ff.) m.E. zu relativieren: Jedenfalls in Europa wird eher schon von einer Übermacht deutscher Rechtswissenschaftler in internationalen Netzwerken gesprochen und scheint mir auch eine Entwicklung der Generationen unverkennbar. Unter den Rechtswissenschaften in allen großen Mitgliedstaaten scheint mir die deutsche (und vielleicht italienische) am stärksten international vernetzt und dies noch zunehmend. Verglichen mit den USA ist schlicht eine andere Perspektive zu konstatieren: Während Rechtswissenschaftler aus den USA in der Tat international sehr präsent erscheinen, liegt doch das Schwergewicht auf dem „Export“ US-amerikanischer Ideen. Umgekehrt ist in den großen Mitgliedstaaten, auch Deutschland, ein stärker ausgeglichenes Bild von „Geben und Nehmen“ prägend. Richtig ist freilich, dass Europäisierung und Internationalisierung noch 
weiter vordringen. Richtig ist umgekehrt freilich auch: Wer sich in Deutschland heute in den Rechtswissenschaften international aufstellen will, hat alle Voraussetzungen dafür - solch eine Profilierung mag nur karrieremäßig noch zu schwach ins Gewicht fallen.

\section{Zu den Rahmenbedingungen für Internationalisierung und Diversität in der Lehre}

Ebenfalls für die Forschung, jedoch vor allem für die Lehre ausgeführt werden die beiden oben genannten Hauptpetita bzw. -stoßrichtungen, auf die hier auch näher eingegangen werden soll. Der Rat konstatiert, dass an juristischen Fakultäten im Durchschnitt ungleich weniger ausländische Dozenten lehren als an anderen Fakultäten (S. 42-44). Das hängt sicherlich auch damit zusammen, dass Recht zwar zunehmend auch Europäisch und International ist (unten IV. 1.), jedoch in fast keinem Rechtsgebiet nicht auch national. Wenige Fächern können ohne fundierte Ausbildung auch im deutschen Recht gelehrt werden. Jedenfalls jedoch sollten Alternativen bedacht werden: In Zeiten knapper Ressourcen kann auf verstärkte Präsenz ausländischer Dozenten in Deutschland gesetzt werden, mit gleichem Recht jedoch auf die Förderung der Sprachfähigkeit und Mobilität der Studierenden. So wird an der Humboldt-Universität fremdsprachiger Rechtsunterricht (durch ausländische Dozenten) für praktisch alle großen Jurisdiktionen angeboten und (fast) verpflichtend gemacht - um dann die Studierenden sehr zu ermutigen ins Ausland zu gehen, etwa im Rahmen des Erasmusprogramms, vor allem jedoch durch Anrechnung ausländischer Studienabschnitte auf den (inländischen) Universitätsteil des Examens (mit heute vier Partneruniversitäten in Genf, London, Paris und Rom). Eine dieser Optionen nehmen fast $20 \%$ der Studierenden wahr - ganz unabhängig von späteren Auslandsstudium zum Master. Und der Prozentsatz derjenigen, die zu den Entscheidungseliten gehören und das Recht wirklich „bilden“ werden, ist kaum höher. Der Finanzierungsetat für das gesamte Bündel (Dozenten, Betreuung etc., ohne Stipendien) erreicht nicht einmal den eines einzigen Lehrstuhls. Zudem mag der Auslandsaufenthalt sogar das „Original“ für Diversitätserfahrungen bilden, der ausländische Dozent nur den Ersatz. Der im Hinblick auf die Dozentenherkunft „internationalste“ (große) Mitgliedstaat, Großbritannien, ist bei der Studierendenmobilität in der Tat weit hinten. Jedenfalls jedoch sollte in Alternativen gedacht werden. Zu Recht weist der Wissenschaftsrat darauf hin, dass gerade für Grenzüberschreitung exzellente Fördermöglichkeiten bestehen, um die Deutschland weltweit beneidet wird. Das gilt es zu erhalten.

\section{Zu den Inhalten}

Am umfassendsten und uneingeschränkt stimme ich dem Wissenschaftsrat in der inhaltlichen Schwerpunktsetzung zu, also in den beiden Hauptpetita und Hauptstoßrichtungen, also in der Frage, in welchen Punkten die - demnach „im Kern gesunde" - Struktur rechtswissenschaftlicher Forschung und Lehre in Deutschland noch am stärksten fortgebildet und gestärkt werden sollte:

Zunächst ist das die Europäisierung und Internationalisierung, die auch Fragen anderer Normtypen und -qualitäten und gesellschaftlicher Umfelder stark in den Fokus rückt („andere Formen der Rechts- und Normbildung“, S. 7). Über 
das zu Sprachfähigkeit und Mobilitätsanreizen Gesagte hinaus sind nur wenige Worte zur Organisation zu nötig: Es existieren mehrfach Doppelabschlüsse einschließlich „Staatsexamens“, teils, wie etwa in Köln oder an der HumboldtUniversität, sogar sowohl in den romanischen als auch in den common law Rechtskreis hinein. Studierende haben also die oben beschriebene Möglichkeit schon heute, letztlich ohne prohibitiven Zusatzaufwand an Zeit (wohl aber bei größerem Engagement). Noch ambitionierter setzt die European Law School (Berlin/London/Paris/Rom) an. Hier werden beide Dimensionen verbunden, mit einem „Staatsexamen“, und je einem vertiefenden, interdisziplinär unterfütterten Master-Jahr in beiden ausländischen Rechtskreisen - und umgekehrt entsprechend für französische, britische und heute auch italienische Studierende. Das dauert jeweils ebenfalls nur fünf, wahlweise sechs Jahre. Alle Studierenden schlossen im klassischen Staatsexamen mit deutlich überdurchschnittlichen Ergebnissen ab. In Sommerschulen steht die Theoriefundierung und Interdisziplinarität eines großen „Rechts- oder Gerechtigkeitsthemas“ im Mittelpunkt. Enge Netzwerke und Freundschaften entstehen. Und ein Kreis von Sponsoren lädt regelmäßig zu Praxisübungen und Praktika ein.

Europäisierung und Theoriefundierung sowie Interdisziplinarität gehen inhaltlich zusammen - im curriculum der European Law School, jedoch auch allgemeiner -, weil ein Recht im Werden, ein Recht, das Grenzen (auch von Rechtsprechungssystemen) überspringt, gar nicht dogmatisch - etwa unter gängigem Verweis auf höchstrichterliche Rechtsprechung - verstanden und vermittelt werden kann, sondern nur im theoretisch und interdisziplinär unterfütterten Diskurs. Nur er kann Akzeptanzbasis über Grenzen sein. Hier prägt die internationale Materie den interdisziplinären Stil!

Welche Lehren sind hieraus zu ziehen? Berlin und die Humboldt-Universität machten es leicht(er), die European Law School zu etablieren. Dennoch steckt viel Zeit und Acquise in dieser heute bestehenden Institution. Andernorts wäre ein mehr an öffentlichem Beitrag zur Ermöglichung hilfreich. Wichtig wäre, so viel zu ermutigen und so wenig zu „prüfen“ wie möglich, konkret: Die Internationalisierung würde enorm gefördert, würde jeder juristischen Fakultät ein Etat (ohne weitere Prüfung) eingeräumt, um einen strukturierten Auslandsstudiengang auf soliden Füßen einzurichten, für 10 Jahre (nach meiner Erfahrung) insgesamt 1 Mio. $€$ je Studiengang - 60 Mio. € über 10 Jahre, 6 Mio. Euro pro Jahr. Juristische Fakultäten sind in der Lage, das eine „richtige“ Projekt auszuwählen. Als „Gegenleistung“ könnte etwa angesetzt werden, dass der oder die Hauptverantwortliche während der drei Aufbaujahre vom Lehrdeputat her wie ein Dekan behandelt wird oder ein zusätzliches Freisemester erhält.

Das vielleicht Wichtigste zuletzt, weil gerade auch die Zuspitzung m.E. zentral ist: Der Wissenschaftsrat beklagt zu Recht nicht so sehr Schwächung und Fehlen von Grundlagenfächern und -veranstaltungen - sie existieren durchaus, haben nur zu wenig Zuspruch und vielleicht auch zu geringes Gewicht im curriculum. Vielmehr beklagt er primär die "dysfunktionale Gegenüberstellung von dogmatischen und Grundlagenfächern einerseits sowie von theoretischer und anwendungsbezogener Forschung andererseits“, und er plädiert insoweit für (mehr) „,wechselseitigen 
Austausch, [der] ... zu Synergieeffekten führen würde ..." (S. 7, 40 f.), insbesondere jedoch in der Lehre für einen Verbund, der in der Tat originell und grds. neu wäre (S. 58 ff.): „Grundlagen- und dogmatische Fächer [sollten] zukünftig verstärkt integrativ und nicht additiv vermittelt werden ... [und dies] muss sich durch das gesamte Studium ziehen." Dem ist umfassend zuzustimmen. Es mag richtig sein, Recht von Anfang an als ein Mittel sozialer Regelung und Gestaltung zu lehren, nicht erst „theoriefreies“ Grundwissen. Recht wird nach meiner Erfahrung nicht zuletzt dann zum fesselnden Gegenstand schon anfangs, wenn es als ein langfristiges Gestaltungsmodell für Gesellschaft und damit als integrativer Teil der Gesellschaftswissenschaften verstanden wird.

Dass hierfür der Schwerpunktteil des Studiums noch mehr Raum gewährt, liegt nahe. Hier kann dann in der Tat auch nicht nur exemplarisch, sondern systematisch und vertiefend gearbeitet werden. Dabei bleibt der Wissenschaftsrat freilich nicht stehen. Er betont, dass es drei methodische Verschränkungsmöglichkeiten gebe, die alle genutzt werden müssten: zwischen (rechts-)theoretischen Grundlagen und Anwendung, zwischen interdisziplinären Grundlagen sowie Erklärungsmodellen (von der Rechtstheorie häufig kaum zu trennen) und Anwendung, und die Verschränkung im Lösungs-, vor allem im Rechtsvergleich.

Dahinter steht, ohne dass der Rat dies explizit machen würde, auch ein spezifisches Bild von Rechtswissenschaft - jedenfalls kann dieses beim vorliegenden Programm besonders gut entwickelt werden: Theoriebasierung und Interdisziplinarität können einerseits dahin gehen, dass vor allem eine Nachbardisziplin oder theoretische Ausrichtung heran- und einbezogen wird, teils sogar dominant. Die ökonomische Analyse des Rechts, die ihren Erfolg auch der Klarheit in der „Anwendung“ auf das Recht verdankt, bildet hierfür ein Beispiel. Und ein Bezug auf diese liegt in wirtschaftsrelevanten Gebieten nahe. Theoriebasierung und Interdisziplinarität können freilich umgekehrt auch so entwickelt werden, dass Recht in der Mitte steht und alle relevanten Disziplinen dazu in Bezug gesetzt werden: etwa soziologische, systemtheoretische, verhaltenswissenschaftliche, evtl. philosophische Überlegungen zum Recht etc. Das Recht ist Gegenstand vieler Disziplinen, die Rechtswissenschaften sollten sie m.E. alle hören. Freilich stößt diese breite - ja umfassende - Interdisziplinarität - jedenfalls für den Einzelnen - an natürliche Grenzen. Gerade in dieser Hinsicht ist es ungemein hilfreich, vom konkreten Problem oder Rechtsgebiet auszugehen. Hier kann dann das in der Rechtswissenschaft reiche - Wissen um Regelungsfragen und -interessen ungleich gezielter verbunden werden mit den jeweils für dieses Gebiet wichtigsten Theorieansätzen und Nachbardisziplinen. Aus eigener Erfahrung würde ich hinzufügen: Es ergeben sich immer wieder neue Einsichten durch solch eine auch innerhalb der Interdisziplinarität Grenzen überschreitende Sicht. Vieles wird bei solch breiter Interdisziplinarität erst ein Versuch sein. Entsteht jedoch ein Markt solcher Gegenüberstellungen und Diskurse, kann dies für die Rechtswissenschaften überaus fruchtbar sein. Wie gesagt: primär wohl jeweils fokussiert auf ein engeres Feld! Das Bild der Rechtswissenschaften wäre dann so zu resümieren: Recht steht im Zentrum, alle Beiträge dazu sind von Interesse. 
Der Wissenschaftsrat analysiert auch, welche Schritte zu tun sind, um diese Ausbildung zu ermöglichen. Er plädiert für Reduktion des sonstigen Stoffes. In Berlin - wo ich lehre - ist der Fächerkanon, der im Staatsexamen geprüft wird, schmaler als in Bayern - wo ich lernte -, vielleicht werden umgekehrt auch Fälle geprüft, die in diesem engeren Spektrum die Fähigkeit, sich auf neue Fragen einzustellen, besonders herausfordern. Jedenfalls würde ich die Umsetzungsmöglichkeiten eher günstiger sehen als der Rat. Auch ohne Reduktion des Stoffes im sonstigen Studium erschiene mir das Jahr der Schwerpunktausbildung gut bemessen, um etwa Kerninstitutionen des Privat- oder des öffentlichen Rechts herauszugreifen und vertiefend nach allen drei (vom Rat) genannten Dimensionen zu durchleuchten. Ebendies bestimmt schon heute das (einjährige) curriculum für diejenigen Studierenden, die in der Spezialisierungsphase im Rahmen der European Law School nach Berlin kommen: Sie lernen dann zum Vertrag - weil sie vom Ausland kommen - das deutsche Vertragsrecht, parallel jedoch: (i) die ökonomische Theorie des Vertrages und Vertragsrechts, (ii) das vergleichende Vertragsrecht, (iii) Europäisches Vertragsrecht in seiner Verschränkung mit dem Marktregulierungsrecht. Im Schwerpunkt wäre ebenso viel Raum. Entsprechendes gilt für das Unternehmens- oder Verfassungsrecht. Es könnte ein Schwerpunktbereichsprogramm unschwer konzipiert werden, in dem zwei oder drei große Institutionen entsprechend vertieft aus vielen Perspektiven durchleuchtet werden. Und diese Ausbildung müssten nicht einmal alle Studierenden durchlaufen - also auch nicht alle Schwerpunkte diesen Zuschnitt erhalten.

Schwerpunkte, die eher der Vermittlung weiteren Stoffwissens dienen, könnten neben solchen (neuen) bestehen bleiben, in denen diese grundlagenorientierte Sicht für einige Kerngebiete und -institutionen des Rechts vermittelt wird. 10-20\% der Studierenden werden sich m.E. für Letztere entscheiden, häufig die Besten. Wieder gilt: Der Prozentsatz derjenigen, die zu den Entscheidungseliten gehören und das Recht wirklich „bilden“ werden, ist sicher nicht höher. Entscheidend ist - wie vom Wissenschaftsrat zu Recht betont - die Vernetzung, d.h. die Bezugnahme der theorieorientierten Ausbildung auf die konkreten rechtlichen Institutionen, etwa das Vertragsrecht. Also nicht Gerechtigkeit abstrakt, sondern Gerechtigkeit spezifisch im Vertragsverhältnis und noch spezifischer: in verschiedenen Vertragstypen und situationen ist in solch einem Ansatz zu erörtern.

Wenn eine Integration schon in die Grundkurse und - vertieft - in die Schwerpunktbereiche möglich erscheint und insoweit neue Brücken gar nicht gebaut werden müssen (auch nicht durch Streichung bisher gelehrter Fächer), so tut sich umgekehrt doch eine Lücke auf, die zu schließen wäre, obwohl sie der Wissenschaftsrat nicht benennt. Es gibt praktisch kaum Bücher, die Dogmatik und Theorie, etwa interdisziplinäre Theorie miteinander verbinden. Das Deliktsrecht von Kötz und Wagner bildet eine der Ausnahmen. Schuld-, Sachen- oder Erbrechtsehrbücher sind dogmatisch und im Wesentlichen frei von den drei genannten Dimensionen. In Italien hat Rodolfo Sacco solch ein vieldimensionales (Schuldrechts-)Lehrbuch vorgelegt, in Großbritannien Hugh Collins ein Vertragsrechtslehrbuch. Doch auch außerhalb Deutschlands sind das seltene Ausnahmen. Der Autor der vorliegenden Zeilen ist gar dafür angegriffen worden, dass er in eine „dogmatische“ Darstellung des Europäischen

(!) Gesellschaftsrechts rechtsvergleichende Abschnitteeinflocht und die Frage 
nach „benchmarks“ aufwarf sowie darüber hinaus auch noch den Stoff mit der relevanten ökonomischen Diskussion und Theorie unterfütterte (Kindler NZG 2011, 1180; Replik NZG 2012, 419). Wäre der Wissenschaftsrat mit seinem zweiten Hauptpetitum erfolgreich, würde das Bild der Rechtswissenschaft fundamental ändern: Was er einfordert - wenn auch nicht ausspricht - sind Lehrbücher, die nicht mehr nur die Dogmatik darstellen, sondern diese mit einer theoretischen, auch interdisziplinären und vergleichenden Perspektive der genannten Art verbinden. Letztendlich würde juristische Ausbildung wieder (zurück) zu einer Ausbildung zu Recht und seiner Rolle in der Gesellschaft mutieren. Der Autor dieser Zeilen wird in Jahresfrist eine nach diesen Grundsätzen konzipierte - und über viele Jahre in Seminaren hinweg gewachsene - Zusammenstellung und Kommentierung großer „Privatrechtstheorien“ vorlegen - vor allem bezogen auf Vertrag, Markt, Risiko und Delikt sowie Unternehmen, aber auch Normsetzung und Gerechtigkeitsfragen des Privatrechts allgemein.

\section{Fazit}

Vier Punkte gilt es zusammenfassend hervorzuheben: Für die Rechtswissenschaften ist eine verlässliche Grundausstattung für eine hinreichend große Zahl von Rechtswissenschaftlern und Rechtswissenschaftlerinnen unverzichtbar und zu verteidigen - vom Wissenschaftsrat durchaus gesehen, vielleicht jedoch noch dezidierter zu betonen. Da steht Deutschland im internationalen Vergleich gut da, dieser „Vorsprung“ sollte erhalten bleiben, wenn internationale Exzellenz ein Ziel sein soll. Und für die Rechtswissenschaften würde ich das „Staatsexamen“ noch dezidierter verteidigen als der Wissenschaftsrat. Denn m.E. können die sinnvollen Fortentwicklungen - in Internationalisierung, jedoch auch in theoretischer und interdisziplinärer Fundierung von rechtswissenschaftlicher Forschung und Lehre - in diesen Rahmen gut integriert werden und dieser Rahmen verbürgt zugleich hochwertige Ausbildung und gute „benchmarks“. Für die Internationalisierung haben die Studierenden der European Law School sowie vieler bilateraler Programme, die auch zum „Staatsexamen“ führen, eindrucksvoll den Beweis erbracht: Sie erzielen Spitzenergebnisse bei zugleich stark internationalisierter Ausbildung. Dieses „volle“ Programm muss auch nicht notwendig jeder Jurastudent durchlaufen, wohl aber ein erheblicher Teil derjenigen, die Recht in der Gesellschaft in Zukunft „entwickeln“. Für die theoretische und interdisziplinäre Fundierung des Rechts und der juristischen Lehre wäre eine Nutzung des universitären Schwerpunktbereichs unschwer möglich. Insgesamt ist das Gutachten ein "großer Wurf“, weil es die zwei Punkte zielsicher herausstellt, an denen die ansonsten m.E. vorzügliche juristische Ausbildung in Deutschland krankt: Internationale Öffnung und eine stärker vernetzte Interdisziplinarität sind zwei Plädoyers von überragender Bedeutung, die es dauerhaft einzulösen gilt.

Dieser Beitrag ist eine gekürzte Fassung von Stefan Grundmann, "Ein doppeltes Plädoyer für internationale Öffnung und stärker vernetzte Interdisziplinarität", Juristenzeitung 2013, p. 693-697. 